\title{
ENSINO SOBRE PERIOPERATÓRIO A PACIENTES: ESTUDO COMPARATIVO DE RECURSOS AUDIOVISUAL (VÍDEO) E ORAL*
}

Adriana Aparecida Delloiagono de Paula** Emília Campos de Carvalho***

PAULA, A.A.D.; CARVALHO, E.C.C. Ensino sobre perioperatório a pacientes: estudo comparativo de recursos audiovisual (vídeo) e oral. Rev.latino-am.enfermagem, Ribeirão Preto, v. 5, n. 3, p. 35-42, julho 1997.

A finalidade do estudo foi comparar, na informação perioperatória de Enfermagem, os recursos audiovisual (vídeo) $e$ oral. Registrou-se como sujeitos 104 pacientes, sendo 54 e 50 para os grupos oral e vídeo, respectivamente. Concluiu-se que os grupos de pacientes eram homogêneos e que não havia diferença significativa entre os dois recursos utilizados, e que independente do recurso utilizado, as respostas emitidas foram qualitativamente melhores. Porém na informação perioperatória com o vídeo utilizou-se 10 minutos e na oral 45 minutos, sendo que o vídeo impede o verbalismo característico dos métodos expositivos e reduz o tempo gasto no oferecimento das informações.

UNITERMOS: ensino sobre perioperátorio, recurso audiovisual (video) e oral

\section{INTRODUÇÃO}

Considerações sobre ensino a pacientes submetidos a cirurgias

A intervenção cirúrgica pode ser uma experiência emocionalmente difícil para o ser humano. Os indivíduos podem apresentar um estado de tensão, ansiedade ou medo, em decorrência do desconhecido, da anestesia, da mutilação e até mesmo da possibilidade de morte.

SANTOS \& CABERLON (1980) afirmam que a preocupação maior envolvendo o paciente cirúrgico decorre da obscuridade das informações, englobando aspectos inerentes ao procedimento cirúrgico ao qual será submetido.

Vários autores afirmam que a visita préoperatória de Enfermagem tem como algumas de suas principais finalidades: reduzir o nível de ansiedade do paciente, colaborar na recuperação da saúde e prestar assistência qualificada ao paciente que se submeterá a tratamento cirúrgico; e deve oferecer informações que contemplem o detalhamento das ações de Enfermagem desenvolvidas no pré, trans e pós-operatório (período perioperatório), sendo transmitida de forma individual ou em grupo, numa linguagem clara e objetiva, compatível com o nível de escolaridade e compreensão do paciente.

No estudo de DELLOIAGONO et al. (1990a), realizado no mesmo hospital-escola, foi constatado como sendo, na opinião dos enfermeiros participantes da amostra, da competência do enfermeiro da unidade de internação, a atividade de informar destinada aos pacientes pré-cirúrgicos, além de considerado o dia anterior à cirurgia como o melhor momento para esta informação.

\section{Recursos audiovisuais/orais na informação}

A literatura tem evidenciado que a informação perioperatória vem sendo realizada de dois modos: quanto ao número de pessoas envolvidas - individual ou grupal; quanto a forma de exposição - oral ou com recurso audiovisual.

Tradicionalmente, a forma oral tem sido a mais comumente empregada na Enfermagem. Para ser melhor sucedida, esta forma requer a presença ao vivo do profissional, transmitindo as informações, daí envolvendo alguns aspectos, tais como adequado conhecimento do profissional quanto ao conteúdo a ser abordado, habilidade de ensino pelo profissional, disponibilidade

\footnotetext{
* Trabalho desenvolvido para obtenção do título de mestre na área de Enfermagem Fundamental da Escola de Enfermagem de Ribeirão Preto da Universidade de São Paulo

** Enfermeira, Mestre em Enfermagem pelo Departamento de Enfermagem Geral e Especializada da Escola de Enfermagem de Ribeirão Preto da Universidade de São Paulo

*** Professor Associado do Departamento de Enfermagem Geral e Especializada da Escola de Enfermagem de Ribeirão Preto da Universidade de São Paulo
} 
de horário que permita ao profissional utilizar seu tempo no atendimento de tal atividade.

Contrapondo-se a este canal, encontra-se o recurso audiovisual que, através de uma tela de vídeo, poderá trazer melhor compreensão das informações oferecidas, bem como poder ser passível de aplicação por contribuir na diminuição do tempo gasto por quem o aplica. Essa mais recente conquista da tecnologia de comunicação, ou seja, a utilização do vídeo, apresentase relevante na área da educação de pacientes.

Corroborando com tal avanço na utilização do vídeo na educação de pacientes está também o trabalho de GAGLIANO (1988) que realizou um levantamento literário abrangendo 25 estudos, buscando definir a eficácia de tal recurso. Aponta como elementos benéficos: ser um método prático; representar baixo custo no investimento de aquisição do equipamento; baixo custo na produção do videotape versus a grande população atingida; assegurar padrão elevado de entendimento de informações; assegurar a repetição de cenas desejadas; o uso do recurso individual ou grupal.

Uma outra importante vantagem, ratificada por WANDERLEY (1984), é a de que o videocassete é um economizador de tempo. Afirma que com ele é necessária apenas uma quarta parte do tempo que seria utilizado para descrever qualquer coisa oralmente por uma pessoa, além de maior nitidez.

Outros ângulos positivos da utilização do recurso audiovisual, citados por CANDEIA (1981), são: complementam e aperfeiçoam o significado da comunicação oral ou escrita; ajudam a objetivar o abstrato, facilitando sua compreensão; impedem o verbalismo característico dos métodos expositivos; ampliam o campo da metodologia; ensejam a familiarização com meios tecnológicos; são poderosos estimuladores da atenção e da motivação; permitem a obtenção de informações difíceis de serem adquiridas por outros meios, principalmente o que é inobservável no tempo e no espaço ou inabordável por seu tamanho; facilitam a expressão pessoal e fomentam o livre intercâmbio de informação; aumentam a eficácia da aprendizagem, e reduzem o tempo que se gasta nela em até $50 \%$.

Observando a literatura na área da saúde, verificar-se-á importante utilização do recurso audiovisual na educação dos pacientes. Com relação à área de Enfermagem, vemos que esta também tem-se utilizado deste recurso na educação dos pacientes, como por exemplo, utilização do videocassete: envolvendo a prática de enfermagem em pediatria (CURRY \& CULLEN (1990); para constatar a ansiedade de pacientes cirúrgicos (GASKEY, 1987); realizar visitas préoperatórias de Enfermagem a crianças, (DURST, 1990); em um programa educacional, para melhorar a qualidade de vida dos indivíduos com neuroma acústico
(GLAVASSEVICH et al., 1991), para informar familiares de crianças com catéter atrial permanente (AKCASU \& BODENMILLER, 1994); em um programa de preparo ambulatorial para crianças e seus pais, antes do procedimento cirúrgico (ELLERTON \& MERRIAM, 1994).

Os artigos específicos de Enfermagem, anteriormente citados, ao mostrarem as vantagens de utilização do videotape nas informações de enfermagem, tais como facilidade de compreensão e assimilação de informação e redução de tempo gasto para realizar as informações, evidenciam a utilização do vídeo como importante estratégia de ensino-aprendizado na assistência de enfermagem.

Nesse sentido, corrobora-se com o emprego do videotape nas informações perioperatórias de Enfermagem, em especial aos pacientes de ginecologia e obstetrícia, sujeitos do estudo que aqui se propõe. Consideramos a possibilidade de que o videocassete, possa vir a ser utilizado enquanto estratégia de ensino na sistematização da assistência de Enfermagem.

\section{OBJETIVOS}

Buscando operacionalizar a utilização de recurso audiovisual (vídeo) na realização de informação perioperatória pela enfermagem, elaboramos os seguintes objetivos: realizar a avaliação diagnóstica das informações das pacientes, sobre a cirurgia, no período pré-operatório; verificar a mudança do índice de informações posterior à realização do ensino sobre o perioperatório utilizando os recursos audiovisual (vídeo) e oral; identificar a diferença do índice de informações para os dois grupos.

\section{MATERIAL E MÉTODO}

\section{Campo de Estudo}

O estudo foi realizado na Unidade de Ginecologia e Obstetrícia do Hospital das Clínicas da Faculdade de Medicina de Ribeirão Preto, da Universidade de São Paulo-HCRP que se situa no Campus da USP.

\section{População e Casuística}

A população deste estudo constou dos pacientes submetidos a cirurgias e que contemplaram os critérios prévios estabelecidos.

\section{Procedimentos}

O trabalho foi realizado em três etapas a se observar: 
1) foi feita uma identificação do conhecimento dos itens abordados na informação perioperatória, através de questões, com vista a avaliação diagnóstica do referido conhecimento, sobre a situação cirúrgica;

2) foi realizada a informação perioperatória para cada grupo de pacientes previamente randomizado quanto ao recurso a ser utilizado;

3) após a informação do grupo, foi feita nova identificação do conhecimento dos itens abordados na informação perioperatória, através das mesmas questões anteriores.

\section{Instrumento: Elaboração e Validação}

\section{Avaliação diagnóstica das informações referidas pela paciente}

Para verificação do conhecimento de cada sujeito sobre os itens que compunham a informação perioperatória, foi elaborado um instrumento.

Esse constituiu-se de uma parte onde se anotava as informações relativas aos dados de identificação das pacientes, o diagnóstico, a cirurgia proposta, a realização de cirurgias anteriores, bem como o tipo de informação perioperatória oferecida (oral ou audiovisual).

Em outra parte, foram relacionadas questões abertas que respeitam a sequência do roteiro previamente proposto pelo vídeo e pela informação oral, abordando os seguintes temas: cuidados pré-cirúrgicos, ambientes, anestesia, ato cirúrgico e cuidados pós-cirúrgicos.

\section{Roteiro de informação oral}

Este recurso foi empregado, na segunda fase para um dos grupos de pacientes e norteou a construção do instrumento anterior.

O roteiro orientou a exposição dos temas ao grupo selecionado. Seu conteúdo foi considerado semelhante ao do vídeo, cuja elaboração foi realizada com base em três aspectos: dados apresentados pela literatura, conteúdo que é apresentado aos pacientes da clínica observada (constatado em trabalho anteriormente realizado por DELLOIAGONO et al. (1990b) e nossa experiência pessoal, enquanto enfermeira de uma unidade de internação cirúrgica.

\section{Vídeo: “Orientação perioperatória de Enfermagem"}

Este recurso foi empregado na segunda fase para um dos grupos de pacientes, previamente randomizados.

Trata-se de um recurso audiovisual, com 10 minutos de duração, e que aborda o mesmo conteúdo do roteiro previamente proposto, para a informação perioperatória: cuidados pré-cirúrgicos, ambiente, anestesia, ato cirúrgico e cuidados pós-cirúrgicos.Este material foi elaborado em momento anterior a este trabalho, por DELLOIAGONO et al. (1990b).

\section{RESULTADOS E DISCUSSÃO}

\section{Caracterização dos sujeitos}

A casuística foi constituída por 104 pacientes internadas na Unidade de Internação de Ginecologia e Obstetrícia, do Hospital das Clínicas da Faculdade de Medicina de Ribeirão Preto-Campus-USP, que seriam submetidas a cirurgias programadas no período destinado a coleta de dados e que contemplaram os critérios já apresentados. Destas, 54 pacientes receberam informação oral e 50 pacientes receberam informação com recurso audiovisual (vídeo).

No dimensionamento da casuística do estudo, deparamos com 12 pacientes que não contemplaram os critérios pré-estabelecidos: menor de 18 anos (1); apresentavam diagnóstico médico ou evidências de distúrbio psiquiátrico (7); apresentavam alteração visual e/ou auditiva (3) e não podiam se locomover (1).

Procuramos analisar algumas variáveis que caracterizaram os sujeitos, sendo que para estas variáveis realizamos o teste $\mathrm{x}^{2}$ e verificamos que as duas populações eram proporcionalmente homogêneas (BUSSAB \& MORETTIN, 1986).

Quanto a variável idade, a idade média foi de 43 anos e a grande maioria dos sujeitos $(62,50 \%)$ encontrase na fase da vida adulta, período compreendido entre 27 e 47 anos, considerada como a fase de maior produtividade social.

Para a variável ocupação, obteve-se que 85 pacientes $(81,73 \%)$ possuíam ocupações nãoqualificadas, predominando as atividades de "do lar" $(46,15 \%)$ e "doméstica" $(20,2 \%)$. Para as demais ocupações, 6,72\% eram de nível inferior de qualificação, 9,63\% de nível médio e 1,92\% de ocupações superiores.

Para a variável grau de instrução, constatamos uma maior incidência de pacientes com grau de instrução até o $1^{\circ}$ grau, sendo alta a frequência das que não concluíram este grau, caracterizando um baixo nível de instrução.

Quanto o período de internação até o momento da coleta de dados, constatamos que o de maior frequência, foi o de apenas um dia $(75,96 \%)$.

Através dos antecedentes pessoais: obstétricos e cirúrgicos, procuramos identificar experiências que as pacientes teriam se submetido previamente. Constatamos que na área obstétrica, as experiências vão desde o parto normal, em casa, até o parto cesárea; e na área cirúrgica, as pacientes relatam tal experiência, nas mais diversas áreas cirúrgicas.

Procuramos também identificar os diagnósticos médicos, e cirurgias propostas, para a complementação da informação perioperatória com dados específicos para cada paciente. 


\section{Análise do conhecimento referido pela paciente}

Para realizarmos a avaliação do conhecimento referido pela paciente, procuramos inicialmente, através das respostas obtidas pelo questionário antes e após a informação, verificar o índice de respostas satisfatórias.

Para a resposta ser considerada satisfatória, deveria ter dados mencionados no roteiro pré-estabelecido $\left(1^{\mathrm{a}}\right.$ fase) ou no conteúdo da estratégia selecionada $\left(2^{\mathrm{a}}\right.$ fase) que apresentavam-se de forma similar. Deste modo, avaliamos todas as respostas das pacientes nos dois momentos, ou seja, antes e após a informação. Os escores adotados foram: $\mathbf{1}$ para as respostas julgadas satisfatória e zero para as não satisfatórias. Com estes escores, uma paciente que apresentasse todas as respostas satisfatórias teria no máximo 32 pontos, ou seja o número de questionamentos feitos. Portanto cada paciente poderia apresentar de zero a 32 pontos. Ao se analisar o conhecimento de cada item questionado, os escores poderiam variar de zero a $\mathbf{5 4}$ pontos, para o grupo que recebeu informação oral e de zero a $\mathbf{5 0}$ pontos para o exposto ao vídeo.

Este procedimento foi utilizado nas fases pré e pós exposição à experiência de ensino.

\section{Escores por item questionado para o conjunto de} pacientes de cada grupo

Procuramos analisar os escores alcançados pelos grupos de pacientes por item questionado. Cabe ressaltar que cada grupo, antes e após a informação poderia apresentar uma variação de escores de zero (tudo insatisfatório) a $\mathbf{5 4}$ para o grupo da informação oral ou 50 pontos para o vídeo, sendo estes últimos escores os referentes ao acerto total de cada questão por todos os sujeitos do grupo.

Os escores apresentados pelos grupos de pacientes segundo o recurso de informação pode ser melhor analisado no Quadro 1.

Quadro 1 - Seqüência dos escores alcançados pelos grupos de pacientes por item questionado, segundo o tipo de informação (vídeo ou oral)

\begin{tabular}{|c|c|c|c|c|c|}
\hline \multirow{2}{*}{\multicolumn{2}{|c|}{ Questionamentos }} & \multicolumn{2}{|c|}{ Oral $(n=54)$} & \multicolumn{2}{|c|}{ Video $(n=50)$} \\
\hline & & Antes & Após & Antes & Após \\
\hline 1 & Preparo anterior & 48 & 52 & 44 & 49 \\
\hline 1a & Coleta de sangue & 51 & 54 & 48 & 50 \\
\hline $1 \mathrm{~b}$ & Tricotornia & 48 & 52 & 43 & 44 \\
\hline $1 \mathrm{c}$ & Medicamento noite & 24 & 48 & 18 & 36 \\
\hline 1d & Alimentação véspera & 36 & 49 & 44 & 50 \\
\hline 2 & Banho & 45 & 53 & 41 & 50 \\
\hline $2 a$ & Vestes & 45 & 54 & 46 & 50 \\
\hline $2 \mathrm{~b}$ & Retirada de jóias, prótese dentária & 52 & 54 & 47 & 50 \\
\hline 3 & Transporte Centro Cirúrgico & 40 & 54 & 37 & 50 \\
\hline 3a & Quem leva para Centro Cirúrgico & 44 & 52 & 36 & 48 \\
\hline 4 & Sala de pré-anestesia & 26 & 53 & 22 & 48 \\
\hline 5 & Temperatura Centro Cirúrgico & 30 & 51 & 19 & 46 \\
\hline $5 a$ & Vestes de equipe Centro Cínírgico & 43 & 53 & 38 & 50 \\
\hline 6 & Transporte Sala de Uperaçáo & 17 & 52 & 17 & 47 \\
\hline 7 & Sala de Operaçăo e o que tem & 39 & 51 & 39 & 50 \\
\hline 8 & Tipos arestesia local & 47 & 54 & 47 & 50 \\
\hline प & Anestesia geral & 23 & 30 & 28 & 44 \\
\hline $9 a$ & Como fíca após anestesia geral & 34 & 51 & 35 & 44 \\
\hline 96 & Local ragui e peri & 39 & 52 & 42 & 50 \\
\hline $9 \mathrm{c}$ & Como fica apos raqui e peri & 32 & 51 & 34 & 49 \\
\hline 10 & Onde irá após cirurgia & 41 & 53 & 41 & 46 \\
\hline$\Pi 1$ & Sala de recuperaçäo & 26 & 50 & 29 & 44 \\
\hline 12 & Quem fica junto na recuperaçăo & 33 & 53 & 36 & 47 \\
\hline $12 \mathrm{a}$ & O que faz a pessoa na recuperação & 30 & 53 & 36 & 47 \\
\hline 13 & Quando sai da recuperaçáo & 34 & 51 & 37 & 46 \\
\hline 14 & Onde vai após recuperação & 43 & 53 & 42 & 47 \\
\hline 15 & Transporte para quarto & 49 & 54 & 49 & 50 \\
\hline 16 & O que fazer na dor & 52 & 54 & 48 & 50 \\
\hline 17 & Levantar com auxilio & 47 & 53 & 35 & 48 \\
\hline 18 & Deambulaçăo após cirurga & 43 & 52 & 49 & 50 \\
\hline 19 & Alimentação após cirurgia & 24 & 46 & 25 & 47 \\
\hline 20 & Condçcóes para alta & 54 & 54 & 46 & 50 \\
\hline
\end{tabular}


Diante dos itens, constatamos que o de menor conhecimento foi comum para ambos os grupos (item 6) e abordava o modo de transporte da sala de pré-anestesia para a sala de operação e este mesmo item foi o que mostrou um maior aumento, para os dois grupos, após o fornecimento da informação. Para os demais itens questionados, constatamos um certo conhecimento para os dois grupos.

Quanto os escores mínimos (zeros) e máximos (54 para oral e 50 para vídeo) estabelecidos, concluímos que nenhum grupo apresentou algum item totalmente desconhecido (escore zero). Corroboramos com BITTAR
\& MARIA (1993) que afirmam que muitos pacientes apresentam um nível de conhecimento acerca do perioperatório e nestes casos, a informação tem como objetivo reforçar estes conhecimentos.

Diferenças de escores por paciente no conjunto de informações, segundo o grupo

Os escores obtidos nesta avaliação podem ser visualizados nas figuras seguintes (Figuras 1 e 2), apresentadas segundo o recurso [oral ou audiovisual (vídeo)].

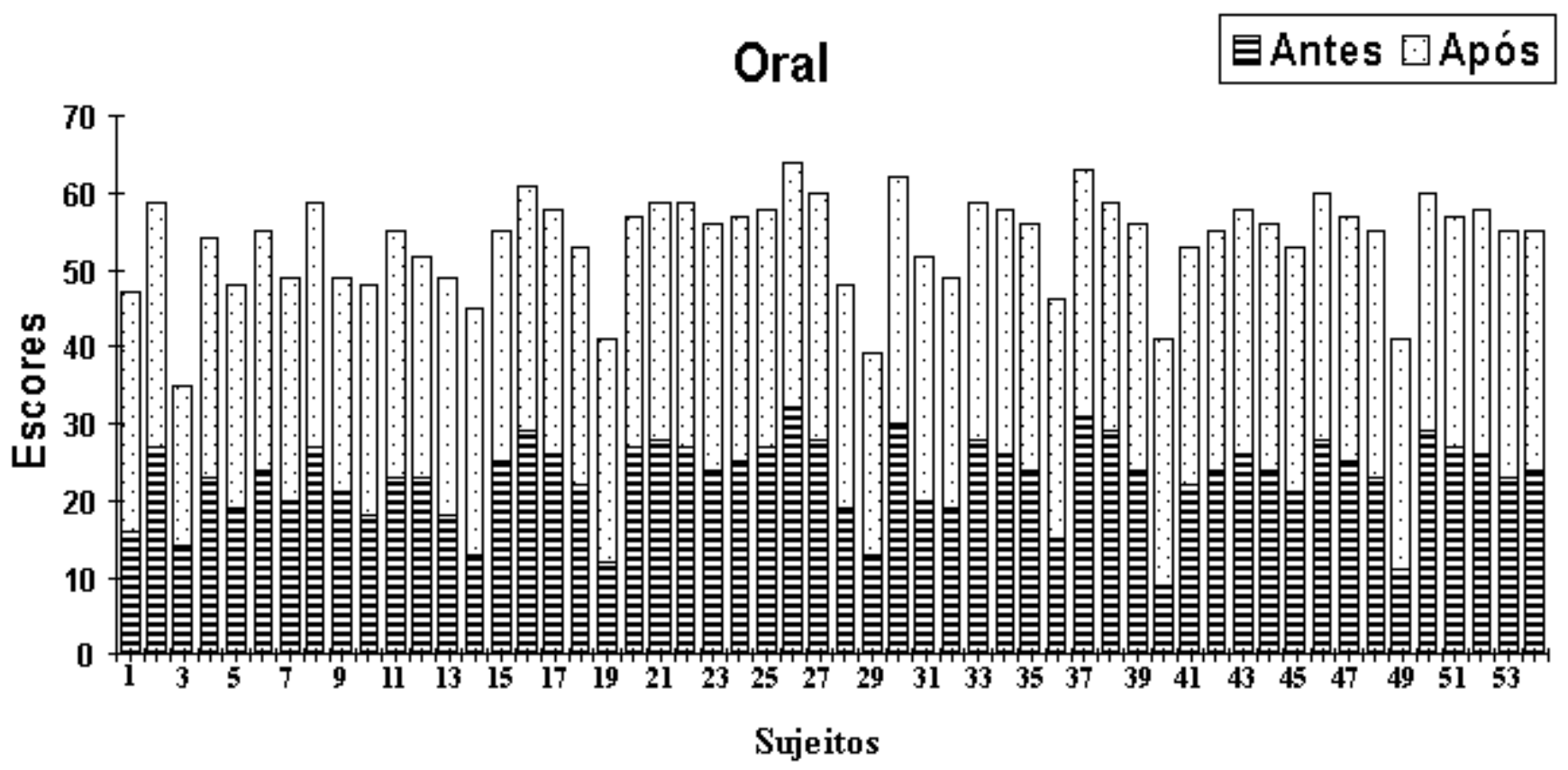

Figura 1 - Diferenças de escores por pacientes antes e após a informação com recurso oral

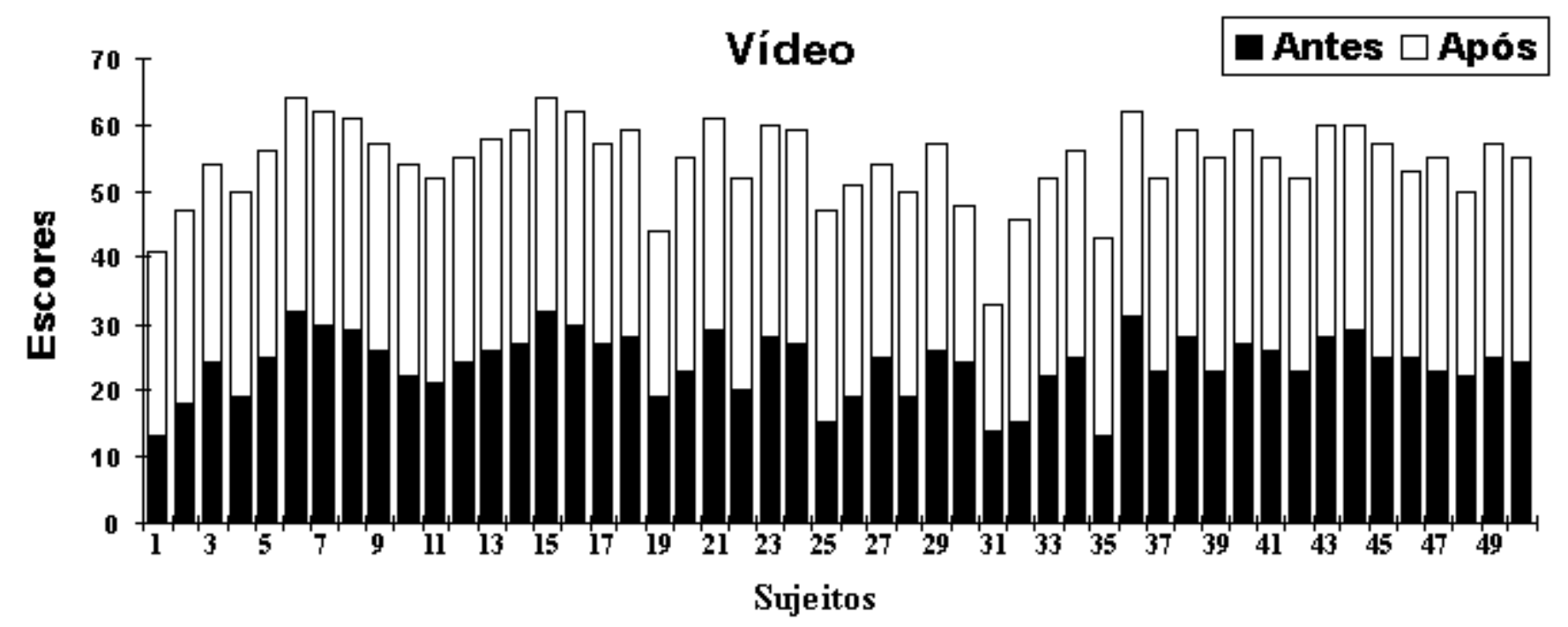

Figura 2 - Diferenças de escores por pacientes antes e após a informação com recurso audiovisual (vídeo) 
Com estes escores, procuramos verificar estatisticamente, através do teste para duas amostras dependentes ou pareadas, se havia diferença nos escores de cada grupo quando da passagem da préinformação para a pós-informação e qual o sentido das diferenças. Em ambos os casos, a hipótese nula da igualdade entre escores foi rejeitada (oral - tcalc $=11,99$; $\mathrm{p}<0,05$, vídeo - tcalc $=10,34 ; \mathrm{p}<0,05)$. Obtivemos então, a mesma conclusão tanto para a utilização do recurso oral, quanto para o recurso audiovisual (vídeo). (BUSSAB \& MORETTIN, 1986).

Constatamos porém, uma diferença qualitativa nas respostas onde o recurso utilizado era o vídeo, pois, muitas pacientes respondiam do mesmo modo que ouviram no filme ou descreviam detalhes das cenas observadas.
Diferenças de escores quanto à qualidade das informações, por paciente, nas fases de pré e pósinformação, segundo o grupo

Procuramos também analisar as diferenças entre respostas antes e após o fornecimento da informação, para cada grupo. Estabelecemos os escores para o segundo momento, ou seja, após a informação, procurando desta forma identificar as respostas que apresentaram crescimento. Ressaltamos novamente que ninguém deixa de ter um conhecimento, mas sim enriquece-o. Assim, atribuíamos o valor 1 para aquelas respostas que mostraram crescimento de conhecimento e o valor zero para as respostas que continuavam com o mesmo conteúdo ou conteúdo inadequado.

A distribuição dos pontos apresentados pelas pacientes, pode ser visualizada na figura a seguir (Figura 3). Destacamos que o objetivo foi comparar os crescimentos dos dois grupos, segundo o recurso empregado.

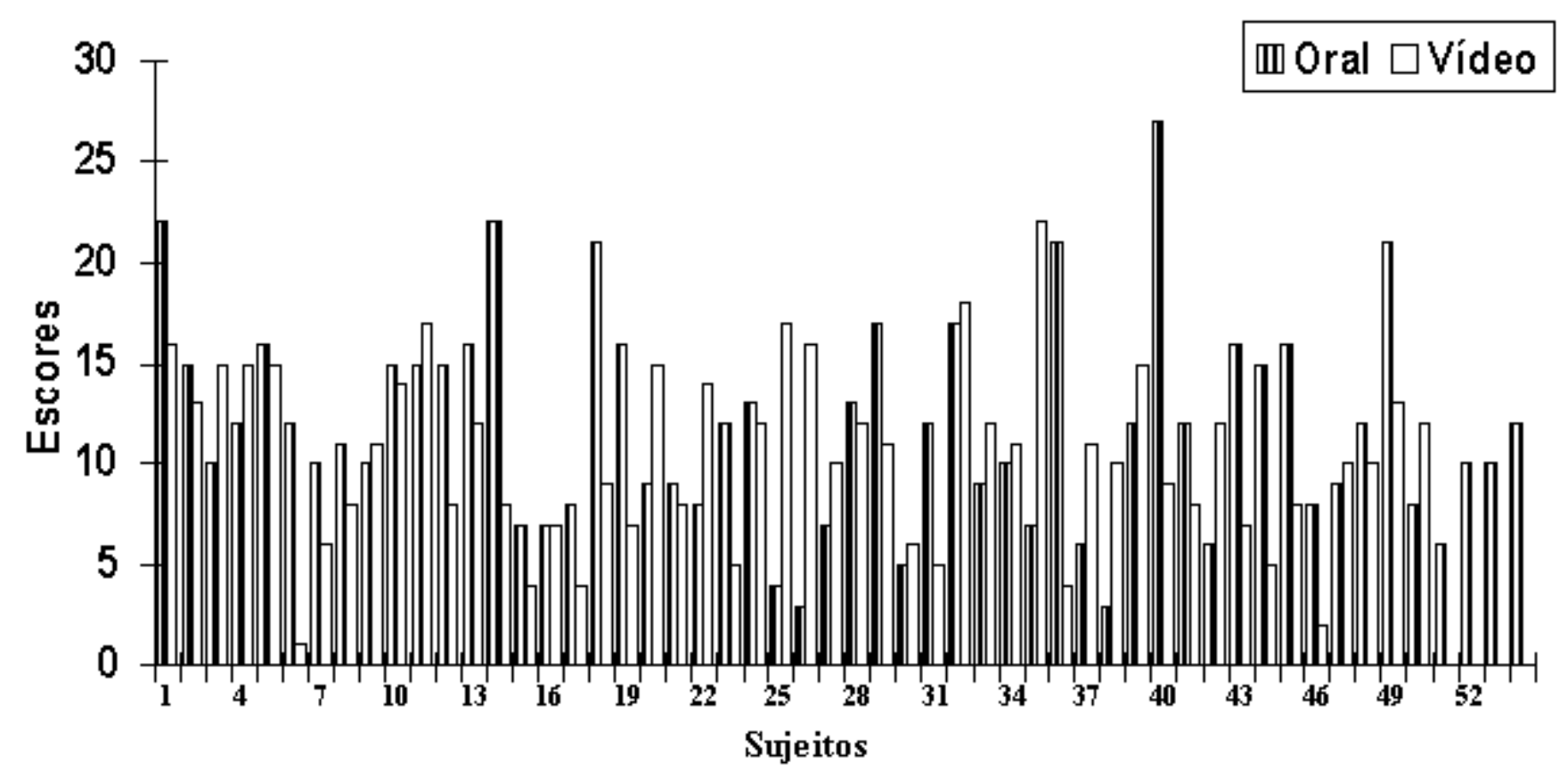

Figura 3 - Diferenças de escores por pacientes antes e apos a realização de todo o procedimento, segundo o recurso de informação

Diante destes valores, ao realizarmos a análise estatística, utilizando o $t$ de Student, concluímos que não há diferenças significativas entre os dois recursos utilizados na informação das pacientes (tcalc $=-1,67$; > 0,05) (BUSSAB \& MORETTIN, 1986).

\section{Tempo utilizado segundo o recurso empregado}

Para a realização das etapas previstas, observamos diferença no tempo gasto, segundo o recurso empregado. Quando o recurso utilizado era o oral, utilizamos na primeira fase, ou seja, na coleta de dados pré-informação cerca de 15 minutos, na segunda fase, quando dávamos as informações, utilizamos 45 minutos e na terceira fase, coleta de dados pós-informação, utilizamos cerca de 40 minutos.

Quando o recurso utilizado era o audiovisual (vídeo), observamos uma variação do tempo na segunda e terceira fases, ou seja, a primeira fase, coleta de dados pré-informação o tempo utilizado foi também cerca de 15 minutos; porém na segunda fase, em que dávamos as informações, o tempo foi de 10 minutos e na terceira fase, 
coleta de dados pós-informações, utilizamos cerca de 15 minutos.

Diante do tempo gasto durante a informação perioperatória corroboramos com CANDEIA (1981) de que com o recurso audiovisual se reduz o tempo gasto na informação em até 50\%.Enfatizamos, com esta variação de tempo gasto, a necessidade de se realizar a informação perioperatória com um instrumento como o que estamos utilizando neste trabalho (vídeo), onde o tempo de informação dos procedimentos seja reduzido, dando condições para a enfermeira atuar diretamente nas necessidades específicas de cada paciente.

\section{CONCLUSÕES}

Diante dos resultados obtidos, concluímos que os grupos de pacientes que receberam informações com diferentes recursos (oral e vídeo) eram homogêneos, não apresentando diferenças significativas entre eles. Isto foi comprovado através da análise realizada quanto às variáveis que caracterizaram os sujeitos do estudo: faixa etária, procedência, ocupação, grau de instrução, bem como experiências cirúrgicas e obstétricas.

Quanto ao conhecimento referido pelas pacientes, na análise, constatamos que o item de menor conhecimento foi comum para ambos os grupos e era o que abordava o modo de transporte da sala pré-anestésica para a sala de operação e este item foi o que mostrou um maior aumento, para os dois grupos, após a informação.

Concluímos que nenhum grupo apresentou algum item totalmente desconhecido (escore zero) e após a informação, vários itens apresentaram um crescimento atingindo o escore máximo.

Acreditamos que com a implementação do recurso audiovisual (vídeo), na orientação de pacientes cirúrgicos, este possa vir a ser utilizado enquanto estratégia de ensino da sistematização da assistência de Enfermagem; com economia de tempo para os profissionais.

Ressaltamos porém a necessidade de complementação de informações aos pacientes quanto aos fatores específicos de sua cirurgia, uma vez que o recurso audiovisual (vídeo) aborda fatores gerais, bem como uma maior atuação sobre as necessidades emocionais de cada paciente.

Consideramos que com a utilização do recurso audiovisual (vídeo), a enfermeira da unidade de internação tenha condições de dar informações de natureza perioperatória aos pacientes e trabalhar as ansiedades destes, uma vez que reduz o tempo de exposição das informações.

\section{TEACHING ON PERIOPERATIVE NURSING: A COMPARATIVE STUDY ON AUDIOVISUAL (VIDEO) AND ORAL RESOURCES}

The aim of this study was to compare information on perioperative Nursing regarding audiovisual (video) and oral resources. 104 patients were registered as subjects and divided in two groups, 54 of them in the oral group and 50 in the video group. Authors concluded that the groups were homogeneous and that there was no significant difference between the two resources used. Independent from the resource used, the responses were qualitatively better. However, regarding perioperative informations, authors spent 10 minutes using the video while oral information lasted 45 minutes. The video unabled verbalization that is characterized by the exposition of methods and reduces the time spent with information.

KEY WORDS: education on perioperative nursing, audiovisual (video) and oral resources

\section{ENSEÑANZA A PACIENTES SOBRE EL PERIOPERATORIO: ESTUDIO COMPARATIVO ENTRE USOS DE RECURSOS}

La finalidad de este estudio fue comparar en la fase perioperatoria de enfermería los dos niveles de aprendizaje, usando recursos (vídeo) y usando solo información oral. Se registraron 104 pacientes de los cuales a 54 se les dio información oral y a 50 se usó vídeo. Se concluyó que los grupos eran homogéneos y que no había diferencias significativas entre los dos métodos utilizados y que independientemente de los recursos las respuestas eran cualitativamente mejores. Pero en la información con vídeo se utilizó 10 minutos y en la forma oral 45 minutos, porque el vídeo impide el verbalismo característico de los métodos expositivos y por ello reduce el tiempo gastado en el ofrecimiento de las informaciones. 


\section{REFERÊNCIAS BIBLIOGRÁFICAS}

01. AKCASU, N.; BODENMILLER, S. Developing and producing a patient education video on care of the permanent right atrial catheter. J. Pediatr. Oncol. Nurs., v. 11, n.3, p. 120-4, Jul. 1994.

02. BITTAR, E.; MARIA, V.L.R. Sistematização da assistência de enfermagem no período perioperatório de um hospital especializado em cardiologia. Rev. Soc. Cardiol. Est. São Paulo., v. 3, n. 2, p.3-7, 1993 Supl. A.

03. BUSSAB, W.O, MORETTIN, P.A. Estatística básica. São Paulo, Ed. Atual, 1986.

04. CANDEIA, J.M. Questionando os audiovisuais. Tecnol.Educ., v. 10, n.40, p. 26-30, maio/jun. 1981

05. CURRY, R.L.; CULLEN, J.C. Using videorecording in pediatric nursing practice. Pediatr. Nurs., v. 16, n. 5., p. 501-4, Sept/Oct. 1990

06. DELlOIAGONO, A.A.; SANTOS, W.D.F.; CARVALHO, E.C. A comunicação enfermeiropaciente na visita pré-operatória. A percepção de enfermeiros assistenciais de um hospital-escola de um município paulista. Medicina, v. 23, n. 1, p. 16, Jan. 1990(a)/Resumo/.
07.

Orientação perioperatória de

enfermagem: conteúdos abordados em situação grupal e a criação de recurso audiovisual Ribeirão Preto, 1990(b)/mimeografado/.

08. DURST, L.M. Perioperative teaching videotape. AORN J., v. 52, n.3, p. 576-84, Sept. 1990.

09. ELLERTON, M.L.; MERRIAM, C. Preparing children and families psychologically for day surgery: an evaluation. J. Adv. Nurs., v. 19, n. 6, p. 1057-62, Jun. 1994.

10. GAGLIANO, M.E. A literature review on the efficacy of video in patient education. J. Med. Educ., v. 63, n. 10, p. 785-92, Oct. 1988.

11. GASKEY, N.J. Evaluation of effect of a pre-operative anesthesia videotape. J. Am. Assoc. Nurs. Anesthet., v. 55, n. 4, p. 341-5, Aug. 1987.

12. GLAVASSEVICH, M.; THOMAS, S.; GALLOWAY, S.C. An educational program to improve quality of life for individuals with acoustic neuroma. $\mathbf{J}$. Neurosc. Nurs., v. 23, n. 4, p. 231-4, Aug. 1991.

13. SANTOS, E.; CABERLON, I.C. Visita pré e pósoperatória aos pacientes. Enfoque., v. 9, n. 6, p. 41-5, dez. 1980.

14. WANDERLEY, C.A. O desafio do uso adequado do videocassete em educação. Tecnol. Educ., v. 13, no 58, p. 27-31, maio/jun. 1984. 